\title{
Incidence of tuberculosis in enlarged neck nodes, our experience
}

\author{
Maharjan $\mathbf{M}^{1}$, Hirachan $\mathrm{S}^{1}$, Kafle $\mathrm{PK}^{2}$, Bista $\mathbf{M}^{2}$, Shrestha $\mathrm{S}^{2}$, Toran $\mathrm{KC}^{3}$, Lakhey $\mathbf{M}^{3}$ \\ ${ }^{1}$ Lecturer, ${ }^{2}$ Assistant Professor, ${ }^{3}$ Associate Professor, Department of ENT, Kathmandu Medical College
}

\begin{abstract}
Objectives: To determine the incidence of tuberculous lymphadenitis in enlarged neck nodes. Materials and methods: Continuous prospective study is carried out in the department of otorhinolaryngology head \& neck surgery, Kathmandu Medical College, Kathmandu, during two years, from January 2006 to January 2008. The study included a group of 155 patients with cervical lymphadenopathy. Each patient underwent a detail clinical Ear, Nose and Throat (ENT) examination and a battery of investigations which included Fine Needle Aspiration Cytology (FNAC) of the nodes, Montoux's test, blood Erythrocyte Sedimentation Rate (ESR) and chest X-ray. Those patients with tubercular lymphadenitis were referred to Directly Observed Therapy System (DOTS) clinic for anti-tubercular therapy. Others with reactive lymphadenitis were treated with antibiotic and those with metastatic neck nodes were treated accordingly.

Results: Of the 155 cases with enlarged neck nodes, 83 (54\%) had tubercular lymphadenitis. Fifty two (33\%) cases had reactive lymphadenitis and $17(11 \%)$ cases were diagnosed with metastatic neck nodes. Fine needle aspiration cytology was found to be highly effective in the diagnosis of tubercular lymphadenitis with $94 \%$ accuracy. Majority of patients were otherwise healthy adults, aged between $8-71$ years. No difference was observed between male and female in this study. Posterior triangle (PT) nodes were most commonly affected group of nodes accounting for 35 (42\%) cases and preauricular region $1(1 \%)$ case being the least commonly affected site. Fifteen (18\%) cases presented with abscess formation. Only $42(50 \%)$ cases had family history of tuberculosis but $8(9 \%)$ patients had previous history of various forms of tuberculosis. Twelve (14\%) patients had positive chest X-ray findings suggesting of concurrent pulmonary tuberculosis. All the patients were referred to DOTS clinic and were treated with category (CAT) - III anti tubercular therapy (ATT). Others with concurrent pulmonary tuberculosis were treated with CAT I regime. None of the patients required surgical treatment.

Conclusion: There is high incidence of tubercular cervical lymphadenitis in patients with enlarged neck nodes in developing countries like Nepal. Involvement of cervical lymphnodes are the most commonly affected group of nodes. Therefore, it is important that otolaryngologists are aware of tuberculosis in the head and neck region.
\end{abstract}

Key words: Tuberculosis, Lymph node, Fine needle aspiration cytology, Lymphadenitis

$\mathrm{T}$ uberculosis is a granulomatous inflammatory process consequent to infection by Mycobacterium tuberculosis (human or bovine type). Tuberculosis affects $1 / 3^{\text {rd }}$ of the world's population. Seventy five percent of the tuberculosis in developing countries is in the economically productive age between $15-50$ years ${ }^{1}$.

Tuberculosis in Nepal is a leading cause of death in adults aged between 15 - 45 years. More than 50,000 new cases are reported every year. About 10,000 adults die of tuberculosis each year in Nepal, making it the leading cause of death among adults ${ }^{1}$. With improvement in economic and social conditions and the use of effective anti-tubercular therapy, the developed nations, and most developing nations, have enjoyed a decline in pulmonary tuberculosis for several decades.
It is now seen that extra-pulmonary presentations form a major proportion of new cases, especially since the advent of the acquired immunodeficiency syndrome epidemic.

Tubercular lympthadenopathy is the most common extra pulmonary form of tuberculosis and cervical lymphnodes are the most commonly affected group of nodes ${ }^{3}$. Therefore, it is important that otolaryngologists are aware of tuberculosis in the head and neck region and its varied manifestations. Fine needle aspiration

Correspondence

Dr. Milan Maharjan

Assitant Professor, Department of ENT

Kathmandu Medical College, Sinamangal

E-mail: puntoo@hotmail.com 
cytology (FNAC) is widely accepted as the accurate, sensitive, specific and cost-effective procedure in the diagnosis of lymphadenopathy. FNAC is reliable as the initial evaluating procedure even in benign disorders; it is also cheap, speedy and easy to perform, with minimum complications, making it suitable for wider application in developing countries with scant resources ${ }^{2}$.

\section{Objectives}

To determine the incidence of tuberculosis in enlarged neck nodes.

Design: Prospective study

\section{Place and duration of study}

This study is carried out in Kathmandu Medical College and teaching hospital, Sinamangal, Nepal. It started from January 2006 till 2008 January, lasting two years.

\section{Materials and methods}

The study included total of 155 patients aged 8 to 71 years, with average age of 39 years. They presented in our out patient's department with complain of enlarged neck nodes persisting for more than two weeks. All the patients underwent thorough ENT examination. Those patients with enlarged lymphnode persistent for more than two weeks were selected for fine needle aspiration cytology (FNAC). Aspiration cytology was done by the expert pathologist with 18 guaze wide bore needle. Smears were made, fixed immediately in ether-alcohol and stained by the Papanicolaou method. Cytological findings like presence of granuloma, casceation necrosis or present of Acid Fast Bacilli (AFB) were considered as positive findings for the diagnosis of tubercular lymphadenitis. Acid fast bacilli smear of the aspirate was done only in suspicious cases. Those patients with cytological findings of tuberculosis underwent battery of investigations which included chest X-ray and three samples of sputum for AFB to exclude pulmonary tuberculosis. Montoux's test and ESR was carried out in all the patients with positive FNA findings. Those with FNA findings suggestive of reactive lymphadenitis were treated with ten days antibiotic therapy and were followed after two weeks to see the size of the node. In majority of patients with reactive lymphadenitis, size of the lymph node resolved following antibiotic therapy but those cases that did not respond to antibiotic therapy underwent repeated FNA to rule out tuberculous cervical lymphadenitis (TBL). After ruling out tuberculosis these patients were kept in close follow up for up to one month duration. Biopsy of the nodes was carried out in patients with persistent enlarged nodes after one month for histopathological confirmation. Those patients with cytological or histopathological confirmed tubercular lymphadenitis were referred to DOTS clinic for anti tubercular therapy (ATT). They were treated according to CAT III regime. All these patients were followed up monthly to see the respond of ATT. Patients with persistent enlarged lymph node after complete course of ATT were considered as multi drug resistance (MDR) case and treated accordingly.

\section{Result}

Out of total 155 cases, 83 (54\%) patients were found to have positive cytological or histological findings suggestive of tubercular lymphadenitis. Fifty two (33\%) cases were diagnosed as reactive lymphadenitis. These cases were treated with two weeks course of antibiotic therapy. Total of $17(11 \%)$ nodes were diagnosed as metastatic lymphnodes, majority are of squamous cell origin, secondary of various head and neck malignancy. Three (2\%) cases were diagnosed as Non Hodgkin's Lymphoma. AFB smear for tubercular bacilli was done only in suspected cases to confirm tuberculosis, which was positive in $32(39 \%)$ cases. FNA results of ten cases were inconclusive and were advised for repeat FNAC after a course of antibiotics as per advice by expert pathologist. Six of these cases had findings consistent with tubercular lymphadenitis. Result of four cases with inconclusive findings underwent biopsy for histopathological confirmation. Out of these four cases, two cases had histological features suggesting reactive lymphadenitis, one case suggesting Non Hodgkin's lymphoma and one case suggesting tubercular lymphadenitis. Majority of patients were otherwise healthy young adults. The age ranged between 8 to 70 years, average age of 39 years. No obvious difference is observed between male and female in the study.

Distribution of lymph nodes, according to MD Anderson's classification in the neck, during the process of development of tuberculous cervical lymphadenitis, posterior triangle (PT) were found to be commonest with $35(42 \%)$ cases, followed by upper deep cervical (UDC) $14(16 \%)$ cases and submandibular (SM) 13 $(15 \%)$ cases. The supra-clavicular (SC) $8(9 \%)$ cases, submental (Sment) 4 (4\%) cases lower deep cervical (LDC) $5(6 \%)$ cases regions, suprasternal region (SS) $3(3 \%)$ cases and preauricular region $1(1 \%)$ case were found to be the least frequently affected. Lymphnode was Unilateral in $71(85 \%)$ cases and bilateral in $12(15 \%)$ cases. Fifteen cases $(18 \%)$ presented with variable degree of abscess formation. In 12 cases combination of repeated wide bore needle aspiration and chemotherapy resolved the nodes. While three cases required combination of abscess drainage and anti tubercular therapy. Surgery was not required in any of the cases.

Of the 83 cases with tubercular lymphadenitis, 42 $(50 \%)$ cases had family history of tuberculosis but only $8(9 \%)$ patients had previous history of various forms 
of tuberculosis. Investigations revealed that 12 (14\%) patients had positive chest X-ray findings suggesting of concurrent pulmonary tuberculosis. Sputum for AFB stain was positive in $11(13 \%)$ cases and ESR was elevated in $66(79 \%)$ cases.
All the patients with isolated tubercular lymphadenitis were referred to DOTS clinic and were treated with CAT III anti tubercular therapy. Others with concurrent pulmonary tuberculosis were treated with CAT I regime.

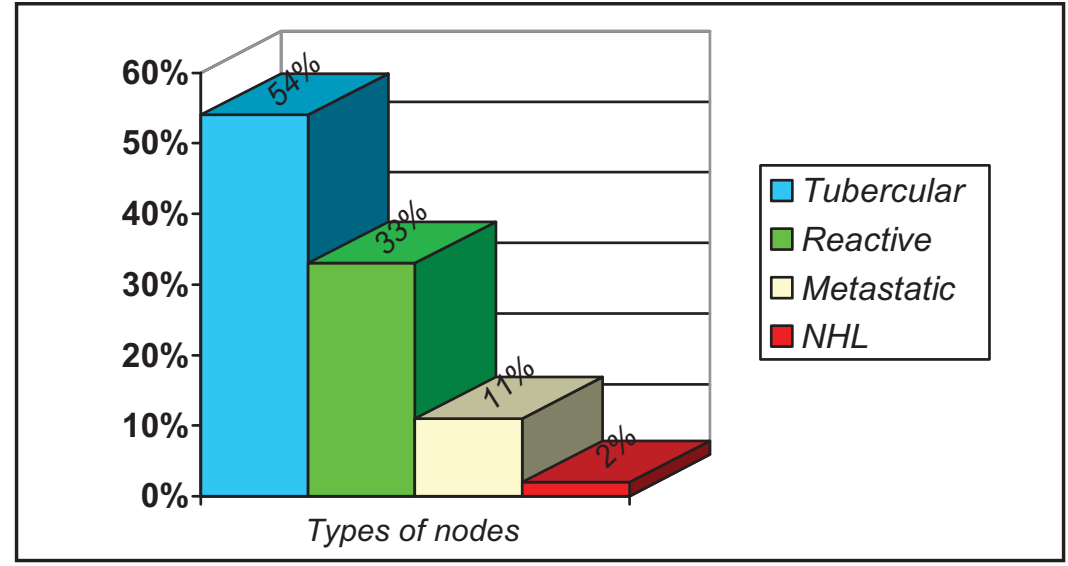

Fig 1: Incidence of various types of lymphnodes

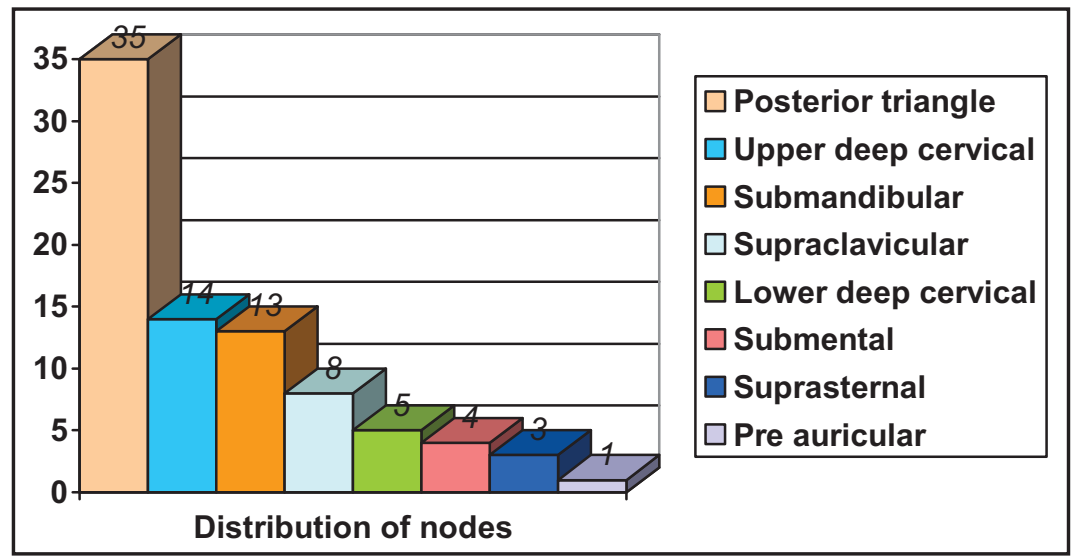

Fig 2: Distribution of lymph nodes in neck

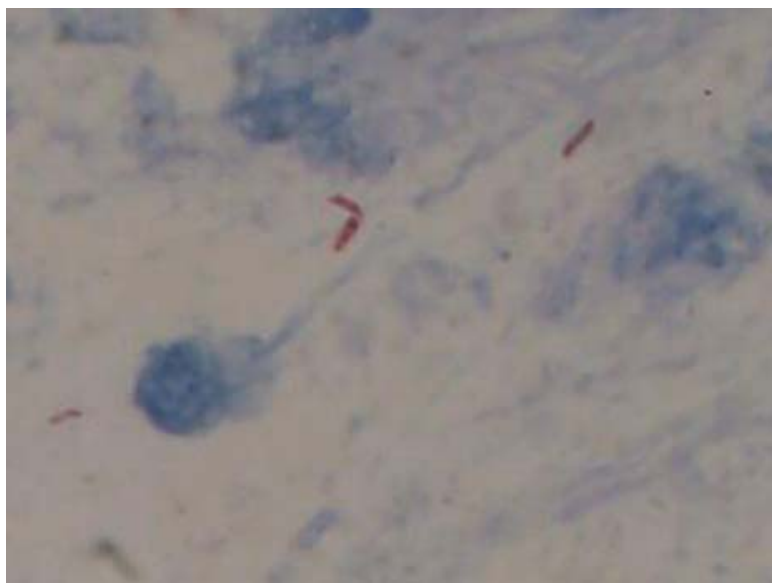

Fig 3: Acid fast bacilli

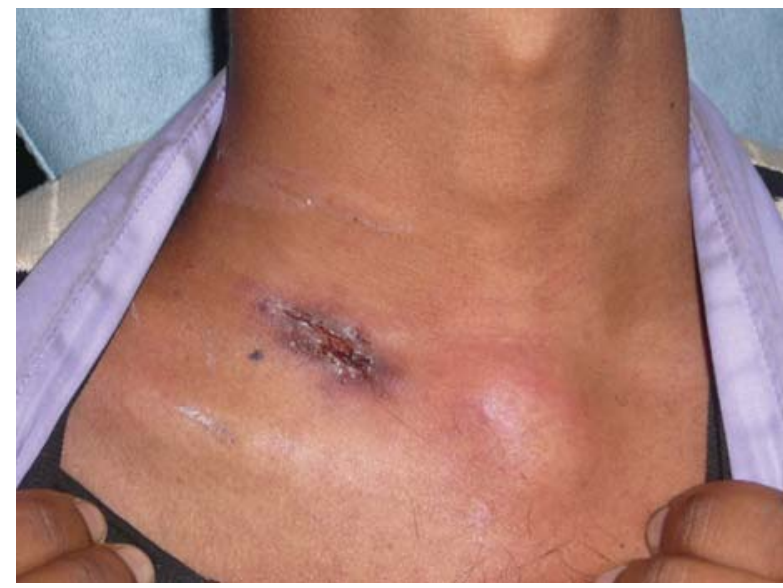

Fig 4: Supraclavicular tubercular fistula with abscess formation 


\section{Discussion}

With betterment in economic and social conditions and the use of effective DOTS program, the developed nations, and most developing nations, have enjoyed a decline in pulmonary tuberculosis for several decades. With advent of the acquired immunodeficiency syndrome epidemic, it is now seen that extra-pulmonary presentations form a major proportion of new cases. Tubercular lympthadenopathy is the most common extra pulmonary form of tuberculosis and cervical lymphnodes are the most commonly affected group of nodes. Sarda et $\mathrm{al}^{2}$ reported very high incidence of tuberculous lymphadenitis in their study, $86 \%$ of the enlarged nodes revealed to be tuberculous in origin. This could be due to good clinical screening of nodes as in our study. Similar high incidence was reported by Dandapat et $\mathrm{al}^{3} 41.5 \%$, Jha et $\mathrm{al}^{4} 63 \%$ and Castro et $\mathrm{al}^{5}$ $46 \%$.

In a study carried out by Haque et $\mathrm{al}^{6}$, the incidence of tubercular lymphadenitis in enlarged neck nodes was very low, $3.5 \%$. This could be because of examination of all the nodes regardless of it's duration of enlargement of nodes, resulting in high incidence of inflammatory features, $87.18 \%$. Similarly Gallesio et $\mathrm{al}^{7}$ reported relatively low incidence, $21 \%$ in his 20 years study.

Male to female ratio is found to be minimal with no obvious preponderance as in other studies like Dandapat et al, Bezabih et $\mathrm{al}^{8}$, and Pandit et $\mathrm{al}^{9}$.

Otherwise healthy young adults are most commonly affected by tuberculous lymphadenitis unlike patients with pulmonary tuberculosis, who are immunocompromised. This was also observed in several other studies like Bezabih et al, Dandapat et al, Pandit et al and Jha et al. But Seth et $\mathrm{al}^{10}$ found it to be equal in all age group except in infants, which is different from all other studies.

In our study involvement of the cervical groups of nodes were found to be the most common, posterior triangle group of node being most commonly affected. Similar result is also reported by Prasad et $\mathrm{al}^{11}$, Baskota et $\mathrm{al}^{12}$, Haque et al, Seth et al and Dandapat et al.

Fine needle aspiration cytology (FNAC) is widely accepted as the accurate, sensitive, specific and costeffective procedure in the diagnosis of lymphadenopathy. Similar experience is observed in our sudy and also in studies carried out by Weiler et $\mathrm{al}^{13}$, Jha et al, AlMullhim et $\mathrm{al}^{14}$, Bezabih et al, and Dandapat et al.

Short term anti tubercular therapy under DOTS is the main line of treatment. Surgery is required in limited cases only, which is supported by Dandapat et al, Jha et al, Weiler et al, Polesky et $\mathrm{al}^{15}$, Ammari et $\mathrm{al}^{16}$ and in our study. Where as Castro et al and Oktay et $\mathrm{al}^{17}$ suggests that surgical excision is the main choice of treatment, which is very different from our study.

\section{Conclusion}

There is very high incidence of tubercular cervical lymphadenitis in patients with enlarged neck nodes in developing countries like Nepal. With improvement in economic, social conditions and the use of effective DOTS program, there is decline in pulmonary tuberculosis in developing countries but there is increase in incidence of extra-pulmonary form of tuberculosis. Lymphnode involvement constitutes the most common presentation of extra pulmonary form of tuberculosis and cervical lymphnodes are the most commonly affected group of nodes. Therefore, it is important that otolaryngologists are aware of tuberculosis in the head and neck region. If the otolaryngologist maintains a high index of suspicion, an early diagnosis can be made with the help of simple investigations.

\section{References}

1. Harries A, Maher D, Uplekar M, Raviglione M. Tuberculosis manual for Nepal, National tuberculosis programme. Ministry of Health, Government of Nepal, 1998 Oct.

2. Sarda AK, Bal S, Singh MK, Kapur MM. Fine needle aspiration cytology as a preliminary diagnostic procedure for asymptomatic cervical lymphadenopathy. J Assoc Physicians India 1990 Mar;38(3):203-5.

3. Dandapat MC, Mishra BM, Dash SP, Kar PK. Peripheral lymph node tuberculosis: a review of 80 cases. Br J Surg. 1990 Aug;77(8):911-2.

4. Jha BC, Dass A, Nagarkar NM, Gupta R, Singhal S. Cervical tuberculous lymphadenopathy: changing clinical pattern and concepts in management. Postgrad Med J. 2001 Mar:77(905):185-7.

5. Castro DJ, Hoover L, Castro DJ, Zuckerbraun L. Cervical mycobacterial lymphadenitis Medical vs surgical management. Arch Otolaryngol. 1985 Dec;111(12):816-9.

6. Haque MA, Talukder SI. Evaluation of fine needle aspiration cytology (FNAC) of lymph node in Mymensingh. Mymensingh Med J. 2003 Jan;12(1):33-5.

7. Gallesio C, De Gioanni PP, Fasciolo A. Tuberculous lesions of the cervico-facial area A case load of 20 year. Minerva Stomatol. 1997 Oct;46(10):507-12. 
8. Bezabih M, Mariam DW, Selassie SG. Fine needle aspiration cytology of suspected tuberculous lymphadenitis. Cytopathology. 2002 Oct;13(5):284-90.

9. Pandit AA, Candes FP, Khubchandani SR. Fine needle aspiration cytology of lymph nodes. J Postgrad Med 1987;33:134.

10. Seth V, Kabra SK, Jain Y, Semwal OP, Mukhopadhyaya S, Jensen RL. Tubercular lymphadenitis: clinical manifestations. Indian J Pediatr. 1995 Sep-Oct;62(5):565-70.

11. Prasad KC, Sreedharan S, Chakravarthy Y, Prasad SC. Tuberculosis in the head and neck: experience in India. J Laryngol Otol. 2007 Oct;121(10):979-85. Epub 2007 Mar 19.

12. Baskota DK, Prasad R, Sinha BK, Amatya RC. Distribution of lymph nodes in the neck in cases of tuberculous cervical lymphadenitis. Acta Otolaryngol. 2004 Nov;124(9):1095-8.

13. Weiler Z, Nelly P, Baruchin AM, Oren S. Diagnosis and treatment of cervical tuberculous lymphadenitis. J Oral Maxillofac Surg. 2000 May;58(5):477-81
14. Al-Mulhim AS, Al-Ghamdi AM, Al-Marzooq YM, Hashish HM, Mohammad HA, Ali AM, Gharib IA. The role of fine needle aspiration cytology and imprint cytology in cervical lymphadenopathy. Saudi Med J. 2004 Jul;25(7):862-5.

15. Polesky A, Grove W, Bhatia G. Peripheral tuberculous lymphadenitis: epidemiology, diagnosis, treatment, and outcome. Medicine (Baltimore). 2005 Nov;84(6):350-62.

16. Ammari FF, Bani Hani AH, Ghariebeh KI. Tuberculosis of the lymph glands of the neck: a limited role for surgery. Otolaryngol Head Neck Surg. 2003 Apr;128(4):576-80.

17. Oktay MF, Topcu I, Senyigit A, Bilici A, Arslan A, Cureoglu S, Yildirim M. Follow-up results in tuberculous cervical lymphadenitis. J Laryngol Otol. 2006 Feb;120(2):129-32. Epub 2005 Nov 25. 